\title{
Two-level frame aggregation scheme under unreliable channel conditions for IEEE
} 802.11n WLANs: a survey

\begin{abstract}
Frame Aggregation schemes defined by IEEE 802.11n is the combination of the Aggregate MAC Service Data Unit (A-MSDU) and MAC Protocol Data Unit (A- MPDU). The units are aimed at maximizing Wireless Local Area Networks (WLANs) efficiency at Media Access Control (MAC) level, via the sharing of headers and timing overheads. Moreover, the combination of A-MSDU and A-MPDU is known as Two-level frame aggregation. In spite of their abilities in improving the throughput of MAC Layer, the scheme is still limited by other overheads as a result of aggregation which affects the system performance. Block Acknowledgement (Block ACK) and Frame aggregation were introduced in order to minimize MAC Layer overheads. Still, there are some parameters that affect the aggregation performance, such as aggregate length, sub frame size and channel condition overheads. AMPDU in other hand minimizes the effect of error condition through sub frame transmission. Therefore, A- MPDU aggregation performances, its limitations, and its promising performances motivated this survey to focus on enhancing Aggregation Mac Protocol Data Unit (eA-MPDU) performance by minimizing the headers overheads of the Two-Level Frame Aggregation Scheme, for the reduction of the channel noise, which by its role will increase the Signal Noise Ratio (SNR) and improve the throughput.
\end{abstract}

Keyword: IEEE 802.11n; Two-level frame aggregation; Overhead; SNR; Unreliable channel; Throughput 